\title{
APPROXIMATING GROUPS OF BUNDLE AUTOMORPHISMS BY LOOP SPACES ${ }^{1}$
}

BY

\author{
ROBERTO BENCIVENGA
}

\begin{abstract}
D. H. Gottlieb proved in 1972 that the group of automorphisms of a numerable $G$-bundle $p: X \rightarrow B$ is weakly homotopy equivalent to $\Omega \operatorname{Map}\left(B, B_{G} ; k\right)$, where $k: B \rightarrow B_{G}$ is a classifying map for $p$. We refine this classical result by constructing a genuine homotopy equivalence between these two spaces which is natural with respect to numerable bundle morphisms, can be generalized to fibre bundles, and can be interpreted as a natural isomorphism between two suitably defined functors.
\end{abstract}

1. Introduction. Let $p: X \rightarrow B$ be a numerable $G$-bundle and denote by $\mathscr{S}(p)$ the group of all $G$-equivariant maps $f: X \rightarrow X$ over $B$ endowed with the compact open topology. This space is of interest not only to mathematicians but also to theoretical physicists $([15,16]$, etc.) who analyze it within the context of the so-called "gauge theories".

While the case where $G$ is abelian (as well as other particular cases $[6,8]$ ) leads to the homeomorphism $\mathbb{S}(p) \simeq \operatorname{Map}(B, G)$, the general case continues to be elusive. The most important result in this area can be considered that of D. H. Gottlieb [6] who showed that there is a weak homotopy equivalence between $\mathscr{G}(p)$ and $\Omega \operatorname{Map}\left(B, B_{G} ; k\right)$, where $k: B \rightarrow B_{G}$ is a classifying map for $p$ and $\operatorname{Map}\left(B, B_{G} ; k\right)$ is the path component of $\operatorname{Map}\left(B, B_{G}\right)$ containing $k$.

The beauty and efficiency of this result leave in the reader the feeling that some of the "weaknesses" of Gottlieb's proof can be polished in order to give rise to a neater statement. We refer here to the fact that Gottlieb uses a Serre fibration (rather than Hurewicz) whose total space is "essentially contractible" (rather than contractible), thus obtaining only a weak homotopy equivalence (rather than a homotopy equivalence). It is also clear, however, that to avoid these problems one has to make some assumptions on the spaces involved. We have chosen to do this by working in the category of $K$-spaces [17], a category large enough to contain all relevant spaces (locally compact spaces, first countable spaces, manifolds, CW-complexes, etc.), but in which the homeomorphism $\operatorname{Map}(X \times Y, Z) \simeq \operatorname{Map}(X, \operatorname{Map}(Y, Z))$ exists for all objects $X, Y, Z$ and, hence, where one can use all the "nice" properties about mapping spaces that one needs. For simplicity we also assume all our spaces to be

Received by the editors March 21, 1983 and, in revised form, November 28, 1983.

1980 Mathematics Subject Classification. Primary 55R10; Secondary 55P35.

Key words and phrases. Bundle automorphism group, loop space, homotopy equivalence, natural isomorphism.

${ }^{1}$ Most of the results presented in this paper were contained in the author's Ph.D. Thesis. 
Hausdorff. With these hypotheses and by using a technique slightly different from that of Gottlieb, we construct a Hurewicz fibration over $\operatorname{Map}\left(B, B_{G} ; k\right)$ with fibre homeomorphic to $(S)(p)$ and with a contractible total space, thus obtaining a homotopy equivalence $\delta_{p}: \Omega \operatorname{Map}\left(B, B_{G} ; k\right) \rightarrow(S(p)$. This method also allows us to relax Gottlieb's condition that $B$ be a $\mathrm{CW}$-complex to requiring only the homotopy type of a CW-complex. Next we show that this construction of $\delta_{p}$ behaves "naturally" with respect to morphisms of numerable bundles, and this leads us to reinterpret Gottlieb's result in categorical terms: more than being simply a map between spaces, $\delta_{p}$ can be viewed as a natural isomorphism between two suitably defined functors.

This naturality also allows us to generalize Gottlieb's result to groups of automorphisms of fibre bundles, even in the case of noneffective action.

The author wishes to express his gratitude to Professors P. Booth, R. Piccinini and D. Sjerve for their cooperation and support.

2. Technical tools from homotopy theory. In this section we recall some standard results of homotopy theory that will be used later and fix the corresponding terminology and notation. For the proofs we refer the reader to the cited or general literature.

Let $p: X \rightarrow B$ be a Hurewicz fibration, $b \in B$ a basepoint, $\iota: F=p^{-1}(b) \rightarrow X$ the inclusion of the fibre, and $x \in F$ a basepoint for both $F$ and $X$. Also assume, from now on, that all spaces denoted by $B$ are path connected.

2.1 LeMMA [13]. If $B$ and $F$ have the homotopy type of a $C W$-complex then so does $X$.

Denote by ev: $P B \rightarrow B$ the path fibration pointed at $b$, by $\Omega B$ its fibre, the space of loops pointed at $b$, and by $c_{b}$ the constant loop at $b$. In the pullback diagram

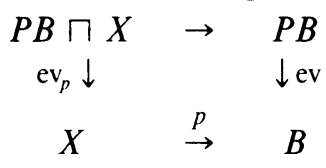

the map $\mathrm{ev}_{p}$ is then a fibration, with the inclusion of the fibre over $x$ being

$$
\gamma: \Omega B \hookrightarrow P B \sqcap X: \ell \leadsto(\ell, x) .
$$

2.2 LEMMA. The map $j: F \rightarrow P B \sqcap X: y \leadsto\left(c_{\ell}, y\right)$ is a homotopy equivalence.

Proof. Apply Theorem 1.2 of [4] to the diagram

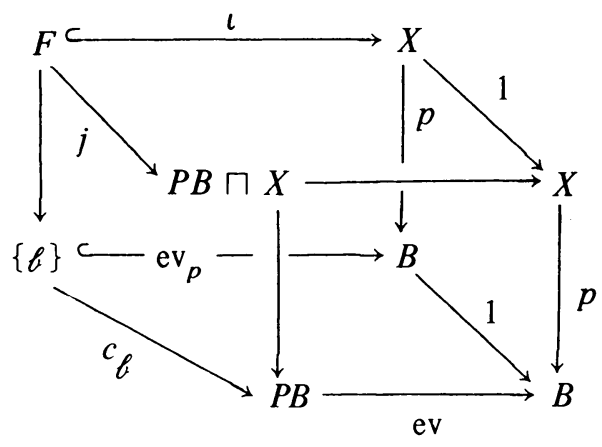


noticing that $c_{\ell}$ is a homotopy equivalence and front and back squares are pullbacks.

Let $\hat{j}$ be a homotopy inverse for $j$ and denote by $\delta$ the composite

$$
\delta=\hat{j} \gamma: \Omega B \rightarrow P B \sqcap X \rightarrow F .
$$

2.3 LEMMA. (a) If $p$ is a homotopy equivalence then $F$ is contractible;

(b) if $X$ is contractible then $\delta$ is a homotopy equivalence;

(c) if $F$ is contractible and $B$ has the homotopy type of a $C W$-complex then $p$ is a homotopy equivalence.

Proof. We only need to notice that for (c) we add Lemma 2.1 to the usual arguments.

2.4 LEMMA. If, in the commutative diagram

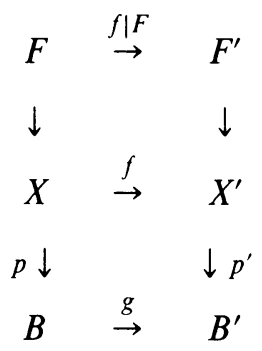

$p$ and $p^{\prime}$ are Hurewicz fibrations and $f$ and $g$ are basepoint preserving, then the diagram

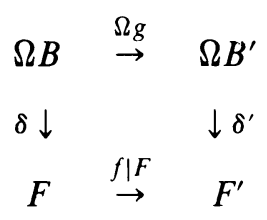

is homotopy commutative.

2.5 Definition. A map $p: X \rightarrow B$ is a numerable $G$-bundle if $G$ is a topological group acting on the right of $X$, there exist a numerable cover $\{U\}$ of $B$ and, for each $U \in\{U\}$, a $G$-equivariant homeomorphism $\varphi_{U}: U \times G \rightarrow p^{-1}(U)$.

2.6 COROLlARY (AFTER [5, TheOREM 4.8]). Every numerable G-bundle is a Hurewicz fibration with fibre $G$.

If $G$ is a topological group we let $p_{G}: E_{G} \rightarrow B_{G}$ denote Milnor's universal numerable $G$-bundle, which is constructed as follows. Consider the set of all sequences $\left\{t_{i}, g_{i}\right\}$, indexed by the natural numbers, such that:

(a) for each $i \in \mathbf{N}, t_{i} \in[0,1]$ and $g_{i} \in G$;

(b) $t_{i} \neq 0$ only for a finite number of indices;

(c) $\sum_{i \in \mathrm{N}} t_{i}=1$.

We introduce an equivalence relation on this set by declaring $\left\{t_{i} g_{i}\right\}$ equivalent to $\left\{t_{i}^{\prime} g_{i}^{\prime}\right\}$ if $t_{i}=t_{i}^{\prime}$ for every $i \in \mathbf{N}$ and, whenever $t_{i}=t_{i}^{\prime} \neq 0$, then $g_{i}=g_{i}^{\prime}$. The resulting quotient set (whose points we still denote by $\left\{t_{i} g_{i}\right\}$ ) is $E_{G}$ and can be 
endowed with a suitable topology which we omit to describe (see [11 or 7]). There is a free action of $G$ on $E_{G}$

$$
\rho_{G}: E_{G} \times G \rightarrow E_{G}:\left(\left\{t_{i} g_{i}\right\}, a\right) \leadsto\left\{t_{i} g_{i} a\right\},
$$

and we let the corresponding orbit space be $B_{G}$, the projection being

$$
p_{G}: E_{G} \rightarrow B_{G}:\left\{t_{i} g_{i}\right\} \leadsto\left\langle t_{i} g_{i}\right\rangle \text {. }
$$

For each $j \in \mathbf{N}$ we can define an open subset $U_{j}^{G}=\left\{\left\langle t_{i} g_{i}\right\rangle \mid t_{j} \neq 0\right\} \subset B_{G}$. The collection $\left\{U_{j}^{G}\right\}$ is a numerable cover for $B_{G}$, and the $G$-equivariant functions

$$
\Phi_{j}^{G}: U_{j}^{G} \times G \rightarrow p_{G}^{-1}(U):\left(\left\langle t_{i} g_{i}\right\rangle, a\right) \leadsto\left\{t_{i} g_{i} g_{j}^{-1} a\right\}
$$

define a locally trivial structure making $p_{G}$ into a numerable $G$-bundle.

2.7 THEOREM (MILNOR [11]). The space $E_{G}$ is contractible, and for every topological space $B$ there exists a bijective correspondence, determined by the pullback construction, between the set of homotopy classes of maps from $B$ to $B_{G}$ and the set of isomorphism classes of numerable G-bundles over $B$.

If $h: G \rightarrow H$ is a continuous homomorphism of topological groups there are induced maps

$$
h^{*}: E_{G} \rightarrow E_{H}:\left\{t_{i} g_{i}\right\} \leadsto\left\{t_{i} h\left(g_{i}\right)\right\}, \quad h_{*}: B_{G} \rightarrow B_{H}:\left\langle t_{i} g_{i}\right\rangle \leadsto\left\langle t_{i} h\left(g_{i}\right)\right\rangle
$$

such that for any $i \in \mathbf{N}$ and $b \in U_{j}^{G}$ the diagram

$$
\begin{array}{ccc}
G & \stackrel{h}{\rightarrow} & H \\
\Phi_{i, \ell}^{G} \downarrow & & \downarrow \Phi_{i, h_{*}(\ell)}^{H} \\
E_{G} & \stackrel{h^{*}}{\rightarrow} & E_{H} \\
p_{G} \downarrow & & \downarrow p_{H} \\
B_{G} & \stackrel{h_{*}}{\rightarrow} & B_{H}
\end{array}
$$

where $\Phi_{i, \ell}^{G}=\Phi_{i}^{G} \mid(\{b\} \times G)$ and $\Phi_{i, h_{*}(b)}^{H}=\Phi_{i}^{H} \mid\left(\left\{h_{*}(b)\right\} \times H\right)$, is commutative.

2.8 LemMa. For any $x \in E_{G}$ and $g \in G, h^{*}(x g)=h^{*}(x) h(g)$; moreover if $h^{\prime}$ : $H \rightarrow K$ is another continuous homomorphism, then $h^{* *} h^{*}=\left(h^{\prime} h\right)^{*}$ and $\left(h_{*}^{\prime} h_{*}\right)=$ $\left(h^{\prime} h\right)_{*}$.

3. Functional bundles. Let $p: X \rightarrow B$ and $p^{\prime}: X^{\prime} \rightarrow B$ be two numerable $G$-bundles and assume the local structures for $p$ and $p^{\prime}$ are determined by the same open cover $\{U\}$ of $B$ (if $B$ is paracompact this can always be arranged). Denote by $\left\{\phi_{U}\right.$ : $\left.U \times G \rightarrow p^{-1}(U)\right\}$ and $\left\{\phi_{U}^{\prime}: U \times G \rightarrow p^{\prime-1}(U)\right\}$ the families of $G$-equivariant local homeomorphisms of $p$ and $p^{\prime}$ respectively. We can then define a set

$$
\left\lceil X, X^{\prime}\right\rceil=\bigcup_{\ell \in B} \mathscr{M}_{G}\left(X_{\ell}, X_{\ell}^{\prime}\right),
$$

where $X_{\ell}=p^{-1}(\ell), X_{\ell}^{\prime}=p^{\prime-1}(\ell)$ and $\mathscr{M}_{G}\left(X_{\ell}, X_{\ell}^{\prime}\right) \subset \operatorname{Map}\left(X_{\ell}, X_{\ell}^{\prime}\right)$ consists of all $G$-equivariant maps. Using the natural projection

$$
\left\lceil p, p^{\prime}\right\rceil:\left\lceil X, X^{\prime}\right\rceil \rightarrow B: \alpha \leadsto p(\operatorname{domain}(\alpha)),
$$


we can endow $\left[X, X^{\prime}\right]$ with a topology by requiring that for each $U \in\{U\}$ the function $\chi_{U}: U \times G \rightarrow\left[p, p^{\prime}\right]^{-1}(U)$, defined by

$$
\chi_{U}(b, g): X_{b} \rightarrow X_{b}^{\prime}: \phi_{U}(b, a) \leadsto \phi_{U}^{\prime}(b, g a),
$$

be a homeomorphism. In this way $\left[p, p^{\prime}\right\rceil$ is a locally trivial map with fibre $G$. The following two lemmas can be easily proved from the definitions.

3.1 Lemma. Let $p: X \rightarrow B$ be a numerable G-bundle and let $q: B \times G \rightarrow B$ be the projection. Then the map

$$
f:\lceil B \times G, X\rceil \rightarrow X: \alpha \leadsto \alpha(\lceil p, q\rceil(\alpha), 1)
$$

is a homeomorphism over $B$.

3.2 Lemma. Let $p: X \rightarrow B$ and $p^{\prime}: X^{\prime} \rightarrow B$ be numerable G-bundles and let $A$ be a subspace of $B$. Then the natural map

$$
\text { ı: }\left[p^{-1}(A), p^{-1}(A)\right] \rightarrow\left[X, X^{\prime} \mid: \alpha \leadsto \alpha\right.
$$

is an inclusion onto $\left[p, p^{\prime}\right\rceil^{-1}(A)$.

For a given map $f: X \rightarrow Y$ we denote by $\sec (f)$ the subspace of $\operatorname{Map}(Y, X)$ consisting of sections to $f$.

3.3 THEOREM. There exists a homeomorphism $\Phi$ between $\sec \left[p, p^{\prime}\right]$ and the space of all G-equivariant maps from $X$ to $X^{\prime}$ over $B$, defined, for given section $s$ and $G$-equivariant map $f$, by the relations

$$
\Phi(s)(x)=s(p(x))(x), \quad \Phi^{-1}(f)(\ell)=f \mid X_{\ell} .
$$

Proof. Combine Corollaries 3.7 and 7.3 of [1] with Theorem 2.1 and Remark 8.2 of [2].

3.4 COROLlary. For any numerable G-bundle $p: X \rightarrow B$ there exists a homeomorphism $\Phi$ between $\sec [p, p \mid$ and $\mathbb{S}(p)$.

Let now $\lambda: G \times F \rightarrow F$ be a (not necessarily effective) action of $G$ on a space $F$ and form the fibre bundle ([7])

$$
p^{F}: X \times_{G} F \rightarrow B:[x, y] \leadsto p(x) .
$$

The group of automorphisms of $p^{F}$, denoted $\mathfrak{F}\left(p^{F}\right)$, is then the group of all maps of the form

$$
f \times{ }_{G} 1: X \times{ }_{G} F \rightarrow X \times{ }_{G} F:[x, y] \leadsto[f(x), y]
$$

where $f \in \mathbb{S}(p)$. By mimicking what we have done for principal bundles, we let $\mathscr{M}_{\lambda}(F, F)$ be the image of the map $\lambda^{*}: G \rightarrow \operatorname{Map}(F, F)$, adjoint to $\lambda$, define a space $F M\left(X \times{ }_{G} F, X \times{ }_{G} F\right)$ consisting of self-maps of the fibres of $p^{F}$, and topologize it by using the canonical injections

$$
\chi_{U}^{F}: U \times \operatorname{Map}(F, F) \rightarrow F M\left(X \times{ }_{G} F, X \times{ }_{G} F\right)
$$


induced by the local structure on $p^{F}$. There is a subspace $\left[X \times{ }_{G} F, X \times{ }_{G} F\right\rceil_{\lambda}$ of $F M\left(X \times{ }_{G} F, X \times{ }_{G} F\right)$ corresponding to the products $\left\{U \times M_{\lambda}(F, F)\right\}$ and a natural projection

$$
\left\lceil p^{F}, p^{F}\right\rceil_{\lambda}:\left\lceil X \times{ }_{G} F, X \times{ }_{G} F\right\rceil_{\lambda} \rightarrow B: \alpha \leadsto p^{F}(\operatorname{domain}(\alpha)) .
$$

The following result is the analogue of Corollary 3.4 for fibre bundles.

3.5 COROLlaRY. There exists a homeomorphism between $\mathfrak{F}\left(p^{F}\right)$ and the image of the $\operatorname{map} \Psi_{*}: \sec \lceil p, p\rceil \rightarrow \sec \left\lceil p^{F}, p^{F}\right\rceil_{\lambda}$ induced by the map

$$
\Psi:\lceil X, X\rceil \rightarrow\left\lceil X \times{ }_{G} F, X \times{ }_{G} F\right\rceil_{\lambda}: \alpha \leadsto \alpha \times{ }_{G} 1 .
$$

Since the map $\Psi$ locally is just $1 \times \lambda^{*}$, we have

3.6 Proposition. The function $\Gamma: \mathfrak{G}(p) \rightarrow \mathfrak{F}\left(p^{F}\right): f \leadsto f \times_{G} 1$ corresponds, under the homeomorphisms of 3.4 and 3.5, to the map $\Psi_{*}$ and is therefore a continuous epimorphism. Moreover it follows from [5, Theorem 4.8] that if $\lambda^{*}: G \rightarrow \mathscr{M}_{\lambda}(F, F)$ is a fibration then so is $\Gamma$.

REMARK. Corollary 3.4 and the fact that $G$ is a topological group can be combined to prove easily that $\mathbb{S}(p)$ is a topological group. Similarly one consequence of Corollary 3.5 is that if $\mathscr{M}_{\lambda}(F, F)$ is a topological group with respect to the group structure induced by $\lambda^{*}$ and the compact open topology, then so is $\mathfrak{F}\left(p^{F}\right)$. This happens, for instance, if $\lambda^{*}$ is an identification (e.g. if $G$ is compact) or if the action is induced by a continuous homomorphism (see $§ 5)$.

4. Constructing a contractible fibre space. Let $p: X \rightarrow B$ be a numerable $G$-bundle and let

$$
\begin{array}{ccc}
X & \stackrel{k^{\prime}}{\rightarrow} & E_{G} \\
p \downarrow & & \downarrow p_{G} \\
B & \stackrel{k}{\rightarrow} & B_{G}
\end{array}
$$

be a pullback diagram, so that $k$ is a classifying map for $p$. The two maps

$$
\begin{aligned}
p \times 1: X \times B_{G} & \rightarrow B \times B_{G}:(x, c) \\
1 \times p_{G}: B \times E_{G} & \rightarrow B \times B_{G}:(b, y) \leadsto\left(b, p_{G}(y)\right)
\end{aligned}
$$

are clearly numerable $G$-bundles, so that we can consider the projection

$$
\left\lceil p \times 1,1 \times p_{G}\right\rceil:\left\lceil X \times B_{G}, B \times E_{G}\right\rceil \rightarrow B \times B_{G},
$$

which, for simplicity, we denote by

$$
\left\lceil p \cdot p_{G}\right\rceil:\left\lceil X \cdot E_{G}\right\rceil \rightarrow B \times B_{G} .
$$

Since the product of two numerable covers is numerable and $\left|p \cdot p_{G}\right|$ is locally trivial over the product cover of $\{U\}$ and $\left\{U_{i}^{G}\right\}$, it follows by Dold's Theorem [5, Theorem 4.8] that $\left[p \cdot p_{G}\right\rceil$ is a Hurewicz fibration. Therefore so are the maps:

$$
\begin{gathered}
\left\lceil p \cdot{ }_{1} p_{G}\right\rceil:\left\lceil X \times E_{G}\right\rceil \stackrel{\left\lceil\cdot p_{G}\right\rceil}{\rightarrow} B \times B_{G} \stackrel{\text { proj }}{\rightarrow} B, \\
\left.\left\lceil p \cdot p_{G}\right\rceil_{B}: \operatorname{Map}\left(B,\left\lceil X \cdot E_{G}\right\rceil\right) \rightarrow \operatorname{Map}\left(B, B \times B_{G}\right): f \leadsto \mid p \cdot p_{G}\right\rceil f .
\end{gathered}
$$


Now notice that the diagram

$$
\begin{aligned}
& \sec \left\lceil p \cdot{ }_{1} p_{G}\right\rceil \quad \hookrightarrow \quad \operatorname{Map}\left(B,\left[X \cdot E_{G}\right\rceil\right) \\
& \theta \downarrow \quad \downarrow\left\lceil p \cdot p_{G}\right\rceil_{B} \\
& \operatorname{Map}\left(B, B_{G}\right) \simeq\left\{\operatorname{id}_{B}\right\} \times \operatorname{Map}\left(B, B_{G}\right) \quad \hookrightarrow \quad \operatorname{Map}\left(B, B \times B_{G}\right)
\end{aligned}
$$

where $\theta$ is the obvious restriction of $\left[p \cdot p_{G}\right]_{B}$, is a pullback, so $\theta$ is a Hurewicz fibration.

4.1 Proposition. If $B$ is homotopy equivalent to a $C W$-complex then the space $\sec \left\lceil p \cdot{ }_{1} p_{G}\right\rceil$ is contractible.

Proof. By Lemmas 3.1 and 3.2 we have for any $b \in B$,

$$
\left\lceil p \cdot{ }_{1} p_{G}\right\rceil^{-1}(\ell)=\left|p \cdot p_{G}\right|^{-1}\left(\{\ell\} \times B_{G}\right) \simeq\left\lceil G \times B_{G}, E_{G}\right\rceil \simeq E_{G},
$$

which is contractible by Theorem 2.7 , hence homotopy equivalent to a $\mathrm{CW}$-complex. By Lemma 2.3(c) it follows that $\left[p \cdot{ }_{1} p_{G}\right]$ is a homotopy equivalence. So the induced map $\left\lceil p \cdot{ }_{1} p_{G}\right\rceil_{B}: \operatorname{Map}\left(B,\left[X \cdot E_{G}\right\rceil\right) \rightarrow \operatorname{Map}(B, B)$ is a fibration and a homotopy equivalence, so its fibre is contractible by Lemma 2.3(a). But such a fibre is $\sec \left\lceil p \cdot{ }_{1} p_{G}\right\rceil$.

In particular, $\sec \left\lceil p \cdot{ }_{1} p_{G}\right\rceil$ is path connected, so its image under $\theta$ must consist of one path component of $\operatorname{Map}\left(B, B_{G}\right)$.

4.2 Lemma. The image of $\sec \left[p \cdot{ }_{1} p_{G}\right\rceil$ under $\theta$ is $\operatorname{Map}\left(B, B_{G} ; k\right)$ and $\theta^{-1}(k)$ is homeomorphic to $B(p)$.

Proof. Let $h: B \rightarrow B_{G}$ be a map and let $s \in \sec \left|p \cdot{ }_{1} p_{G}\right|$. Denote by $\operatorname{gr}(h)$ the graph of $h$ in $B \times B_{G}$ and observe that $(p \times 1)^{-1}(\operatorname{gr}(h))=X$ and $\left(1 \times p_{G}\right)^{-1}(\operatorname{gr}(h))$ $=X^{\prime}$, where $p^{\prime}: X^{\prime} \rightarrow B$ is the numerable $G$-bundle pulled back by $h$. Then the following are equivalent:

$$
\theta(s)=h \Leftrightarrow\left\lceil p \cdot p_{G}\right\rceil s=1 \times h \Leftrightarrow s(B) \subseteq\left\lceil p \cdot p_{G}\right\rceil^{-1}(\operatorname{gr}(h)),
$$

and by Lemma 3.2 the last statement is equivalent to

$$
s(B) \subseteq\left\lceil X, X^{\prime}\right\rceil \Leftrightarrow \Phi(s): X \rightarrow X^{\prime} \text { is } G \text {-equivariant and over } B \text {. }
$$

But by [7, Theorem 3.2] $\Phi(s)$ exists if and only if $p$ and $p^{\prime}$ are isomorphic, that is if $h$ is homotopic to $k$ and, hence, $h \in \operatorname{Map}\left(B, B_{G} ; k\right)$. In particular, $\theta^{-1}(k)=\sec \lceil p, p\rceil$ $\simeq \mathscr{B S}(p)$.

4.3 Theorem. Let $p: X \rightarrow B$ be a numerable $G$-bundle and assume $B$ has the homotopy type of a $C W$-complex. Then there exists a homotopy equivalence $\delta_{p}$ : $\Omega \operatorname{Map}\left(B, B_{G} ; k\right) \rightarrow$ SH $(p)$.

Proof. Apply Lemma 2.3(b) to the Hurewicz fibration $\left.\theta: \sec \mid p \cdot{ }_{1} p_{G}\right\rceil \rightarrow$ $\operatorname{Map}\left(B, B_{G} ; k\right)$.

4.4 Corollary. If $\operatorname{Map}\left(B, B_{G}\right)$ contains two path components having different homotopy groups in at least one dimension, then there exist two distinct numerable $G$-bundles over B having groups of automorphisms the homotopy groups of which are different. 
EXAMPLE. In [9] Koh showed that $\pi_{3}\left(\operatorname{Map}\left(S^{4}, S^{4} ; k\right)\right) \approx \mathbf{Z}_{24 m} \oplus \mathbf{Z}_{12}$, where $m=|\operatorname{deg} k|$. Since every simplicial complex is the classifying space of some topological group [10], we can use this computation to construct examples of numerable bundles over $S^{4}$ for which $\mathscr{S}(p)$ and $\operatorname{Map}\left(S^{4}, G\right)$ do not even have the same homotopy groups. Clearly the same idea can be used in other cases.

ReMARK. If $B_{G}$ is an $H$-group then all path components of $\operatorname{Map}\left(B, B_{G}\right)$ have the same homotopy type [9]. This happens, for instance, when $G$ is an infinite unitary or orthogonal group or their special subgroups (i.e. $G=\mathrm{O}, \mathrm{U}, \mathrm{SO}, \mathrm{SU}$ ).

5. Naturality of the loop approximation. Every continuous homomorphism $h$ : $G \rightarrow H$ between topological groups induces a left action of $G$ on $H$ in the obvious way. Moreover if $p: X \rightarrow B$ is a numerable $G$-bundle it is easy to show that the associated bundle

$$
p^{h}: X \times{ }_{G} H \rightarrow B:[x, a] \leadsto p(x)
$$

is a numerable $H$-bundle, and if the diagram

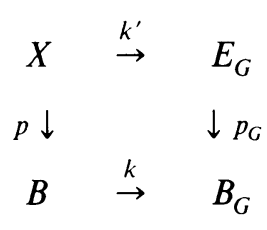

is a pullback, then so is

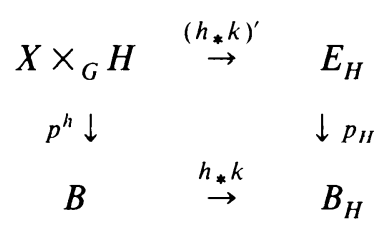

where $\left(h_{*} k\right)^{\prime}[x, a]=h^{*}\left(k^{\prime}(x)\right) a$. In particular, $h_{*} k$ classifies $p^{h}$. Also, as in 3.6, we can prove that there exists a continuous homomorphism $\Pi_{p}^{h}: \mathscr{S}(p) \rightarrow \mathfrak{G S}\left(p^{h}\right)$ : $f \leadsto f^{h}$, where $f^{h}[x, a]=[f(x), a]$. So we can draw a diagram of maps

$$
\begin{array}{ccc}
\Omega \operatorname{Map}\left(B, B_{G} ; k\right) & \stackrel{\delta_{p}}{\rightarrow} & \mathfrak{S}(p) \\
\Omega\left(h_{*}\right)_{B} \downarrow & & \downarrow \Pi_{p}^{h} \\
\Omega \operatorname{Map}\left(B, B_{H} ; h_{*} k\right) & \stackrel{\delta_{\left(p^{h}\right)}}{\rightarrow} & \mathfrak{S}\left(p^{h}\right)
\end{array}
$$

where $\Omega\left(h_{*}\right)_{B}$ is induced from $h_{*}$ by composition and $\delta_{p}$ and $\delta_{\left(p^{h}\right)}$ are as described in Theorem 4.3.

The obvious question now is whether this diagram has commutativity properties. We cannot expect strict commutativity, since $\delta_{p}$ and $\delta_{\left(p^{h}\right)}$ are determined only up to homotopy, but homotopy commutativity will be sufficient to claim "naturality" properties for our construction. 
5.1 LemMa. There is a continuous function over $B, \Psi:\left\lceil X \cdot E_{G}\right\rceil \rightarrow\left\lceil\left(X \times{ }_{G} H\right) \cdot E_{H}\right\rceil$, defined, for a given $\alpha: p^{-1}(b) \times\{c\} \rightarrow\{b\} \times p_{G}^{-1}(c)$ in $\left\lceil X \cdot E_{G}\right\rceil$, by setting

$$
\begin{gathered}
\Psi(\alpha):\left(p^{h}\right)^{-1}(b) \times\left\{h_{*}(c)\right\} \rightarrow\{b\} \times p_{H}^{-1}\left(h_{*}(c)\right) \\
:\left([x, a], h_{*}(\mathscr{C})\right) \leadsto\left(\mathscr{B}, h^{*}(\alpha(x)) a\right) .
\end{gathered}
$$

Proof. For each local homeomorphism $\varphi_{U}: U \times G \rightarrow p^{-1}(U)$ of $p$ and each $i \in \mathbf{N}$ there are, by the definition of the topologies on $X \times{ }_{G} H,\left\lceil X \cdot E_{G}\right\rceil$ and $\left\lceil\left(X \times{ }_{G} H\right) \cdot E_{H}\right\rceil$, local homeomorphisms,

$$
\begin{aligned}
& \chi_{U, i}: U \times U_{i}^{G} \times G \rightarrow\left\lceil\left. p \cdot p_{G}\right|^{-1}\left(U \times U_{i}^{G}\right),\right. \\
& \chi_{U, i}^{h}: U \times U_{i}^{H} \times H \rightarrow\left|p^{h} \cdot p_{H}\right|^{-1}\left(U \times U_{i}^{H}\right),
\end{aligned}
$$

defined by the relations

$$
\begin{aligned}
\chi_{U, i}(b, c, g)\left(\varphi_{U}(b, a), c\right) & =\left(b, \Phi_{i}^{G}(c, g a)\right), \\
\chi_{U, i}^{h}(b, c, g)\left(\left[\varphi_{U}(b, 1), a\right], c\right) & =\left(b, \Phi_{i}^{H}(c, g a)\right),
\end{aligned}
$$

and they are clearly over $B$. Using the definitions of the maps involved and the commutativity of diagram (A), it is not difficult to verify that the diagram

$$
\begin{array}{ccc}
\left|p \cdot p_{G}\right|^{-1}\left(U \times U_{i}^{G}\right) & \stackrel{\Psi}{\rightarrow} & \left|p^{h} \cdot p_{H}\right|^{-1}\left(U \times U_{i}^{H}\right) \\
\chi_{U, i} \uparrow & & \uparrow \chi_{U, i}^{H} \\
U \times U_{i}^{G} \times G & \stackrel{1 \times h_{*} \times h}{\rightarrow} & U \times U_{i}^{H} \times H
\end{array}
$$

is commutative. This suffices to prove the claim.

5.2 Proposition. The map $\Psi_{*}: \sec \left\lceil p \cdot{ }_{1} p_{G}\right\rceil \rightarrow \sec \left\lceil p^{h} \cdot{ }_{1} p_{H}\right\rceil$, induced by $\Psi$, completes the diagram of basepoint preserving maps,

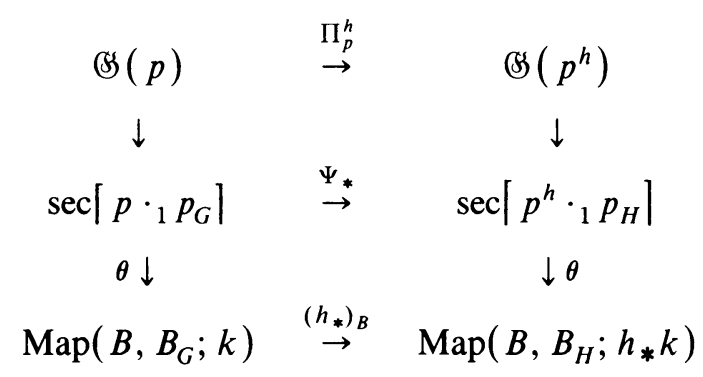

in which the identity automorphisms of $\mathbb{S}(p)$ and $\mathbb{S}\left(p^{h}\right)$ determine the basepoints for the two spaces of sections.

Proof. The diagram used in the proof of Lemma 5.1 shows that if $s \in \sec \left\lceil p \cdot{ }_{1} p_{G}\right]$, $b \in B$ and $s(b)$ is over $(b, c)$, then $\Psi_{*}(s)(b)=\Psi_{s}(b)$ is over $\left(b, h_{*}(c)\right)$ and, thus, that $\Psi_{*}$ induces, between the two mapping spaces, the map $\left(h_{*}\right)_{B}$. This proves the commutativity of the lower square. The commutativity of the upper square can be verified by analyzing the inclusions of $\mathscr{S}(p)$ into $\sec \left\lceil p \cdot{ }_{1} p_{G}\right\rceil$ and of $\mathscr{S}\left(p^{h}\right)$ into $\sec \left\lceil p^{h} \cdot{ }_{1} p_{H}\right\rceil$ as described in Lemma 4.2. 
5.3 Theorem. Let $p: X \rightarrow B$ be a numerable G-bundle classified by a map $k$ : $B \rightarrow B_{G}$ and assume $B$ has the homotopy type of a $C W$-complex. If $h: G \rightarrow H$ is $a$ continuous homormorphism of topological groups, there exists a homotopy commutative diagram

$$
\begin{array}{ccc}
\Omega \operatorname{Map}\left(B, B_{G} ; k\right) & \stackrel{\delta_{p}}{\rightarrow} & (\mathfrak{S}(p) \\
\Omega\left(h_{*}\right)_{B} \downarrow & & \downarrow \Pi_{p}^{h} \\
\Omega \operatorname{Map}\left(B, B_{H} ; h_{*} k\right) & \stackrel{\delta_{\left(p^{h}\right)}}{\rightarrow} & \mathbb{S}\left(p^{h}\right)
\end{array}
$$

where the horizontal maps are homotopy equivalences.

Proof. Apply Lemma 2.4 to the diagram of Proposition 5.2.

6. Fibre bundles. In this section we shall use the techniques and results of the previous sections to generalize Theorem 4.3 to fibre bundles. Let $p: X \rightarrow B$ be a principal $G$-bundle, $\lambda: G \times F \rightarrow F$ be a (not necessarily effective) action of $G$ on a space $F, Q=G / \operatorname{ker}\left(\lambda^{*}\right)$ and $q: G \rightarrow Q$ be the natural projection.

6.1 LeMma. If $\lambda^{*}$ is a fibration then so are $q$ and $\Pi_{p}^{q}$.

Proof. This is as in 3.6.

6.2 LemMa. The group $\mathfrak{F}\left(p^{q}\right)$ is the image of $\Pi_{p}^{q}: \mathscr{H}(p) \rightarrow \mathscr{G}\left(p^{q}\right)$. Moreover if $\lambda^{*}$ is an identification then $\mathfrak{F}\left(p^{F}\right) \simeq \mathfrak{F}\left(p^{q}\right)$.

Proof. The first part is obvious. Now, if $\lambda^{*}$ is an identification, then $Q$ and $\mathscr{M}_{\lambda}(F, F)$ are isomorphic topological groups. We can use this fact and the local structures of $\lceil p, p\rceil,\left\lceil p^{F}, p^{F}\right\rceil_{\lambda},\left\lceil p^{q}, p^{q}\right\rceil_{q}$ to show that there is a diagram

$$
\begin{aligned}
& \lceil X, X\rceil \\
& \Psi_{F} \swarrow \quad \searrow \Psi_{Q} \\
& \left\lceil X \times_{G} F, X \times_{G} F\right\rceil_{\lambda} \quad \stackrel{t}{\rightarrow} \quad\left\lceil X \times_{G} Q, X \times_{G} Q\right\rceil_{q}
\end{aligned}
$$

commutative over $B$ and such that $\Psi_{F}$ induces $\Gamma, \Psi_{Q}$ induces $\Pi_{p}^{q}$ and $t$ is a homeomorphism. This, together with 3.4 and 3.5 , justifies the claim.

6.3 COROLlaRY. If $\lambda^{*}$ is an identification and a fibration then $\mathfrak{F}\left(p^{F}\right)$ is homeomorphic to a complete set of path components of $\left(S\left(p^{q}\right)\right.$.

REMARK. If $G$ is compact, $\lambda^{*}$ is always an identification, and if $G$ is also a Lie group, $\lambda^{*}$ is also a fibration.

By combining these results with Theorems 4.3 and 5.3 we obtain

6.4 TheOREM. Let $p: X \rightarrow B$ be a numerable $G$-bundle classified by $k: B \rightarrow B_{G}$ and assume $B$ has the homotopy type of a $C W$-complex. Let $\lambda: G \times F \rightarrow F$ be an action such that the associated map $\lambda^{*}: G \rightarrow \mathscr{M}_{\lambda}(F, F)$ is an identification and a fibration. Then $\mathfrak{F}\left(p^{F}\right)$ is homotopy equivalent to the subspace of $\Omega \operatorname{Map}\left(B, B_{Q} ; q_{*} k\right)$ consisting 
of all the path components in the image of the homomorphism

$$
\pi_{0}\left(\Omega\left(q_{*}\right)_{B}\right): \pi_{0}\left(\Omega \operatorname{Map}\left(B, B_{G} ; k\right)\right) \rightarrow \pi_{0}\left(\Omega \operatorname{Map}\left(B, B_{Q} ; q_{*} k\right)\right) .
$$

Notice that this is a generalization of Theorem 4.3 since the latter can be obtained by setting $F=G$ and $\lambda^{*}=$ identity.

6.5 COROLlary. Under the same hypotheses of Theorem 6.4 the component of the identity of $\mathfrak{F}\left(p^{F}\right)$ is homotopy equivalent to the component of the constant loop in $\Omega \operatorname{Map}\left(B, B_{Q} ; q_{*} k\right)$ and so, in particular, for $i \geqslant 1$,

$$
\pi_{i}\left(\mathfrak{F}\left(p^{F}\right)\right) \approx \pi_{i+1}\left(\operatorname{Map}\left(B, B_{Q}: q_{*} k\right)\right) .
$$

Proof. We only need to notice that by construction the map $\delta$ sends the component of the basepoint of the fibre $F$ to the component of the constant loop of $\Omega B$.

REMARK. Corollary 6.5 implies that the fundamental and higher homotopy groups of $\mathfrak{F}\left(p^{F}\right)$ depend not on the structure group of the bundle, but on the quotient of this obtained by eliminating all the elements which act trivially. However the same is not true for $\mathfrak{F}\left(p^{F}\right)$ itself. For instance the map

$$
f: S^{1} \times S^{1} \rightarrow S^{1} \times S^{1}:\left(e^{i \pi \theta}, e^{i \pi \rho}\right) \leadsto\left(e^{i \pi \theta}, e^{i \pi(\theta+\rho)}\right)
$$

is an automorphism of the trivial $S^{1}$ bundle $p: S^{1} \times S^{1} \rightarrow S^{1}$ if we consider it as a fibre bundle associated to itself via the identity on $S^{1}$, but not if we consider it as a fibre bundle associated to the trivial R-bundle $p^{\prime}: S^{1} \times \mathbf{R} \rightarrow S^{1}$ via the usual projection $e: \mathbf{R} \rightarrow S^{1}: \theta \leadsto e^{i \pi \theta}$ (if it were one could factor the identity on $S^{1}$ through R). So in this case $f \in \mathfrak{F}\left(p^{\text {id }}\right)$, but $f \notin \mathfrak{F}\left(p^{\prime e}\right)$, although $p^{\text {id }}=p^{\prime e}$.

A refinement of Theorem 6.4 is obtained in the following particular case.

6.6 LEMMA. If $B$ is a $C W$-complex of dimension $n$ and $\operatorname{ker}\left(\lambda^{*}\right)$ is $(n-1)$-connected or if $B$ is an n-connected $C W$-complex and $\pi_{i}\left(\operatorname{ker}\left(\lambda^{*}\right)\right)=0$ for $i \geqslant n-1$, then $\mathfrak{F}\left(p^{F}\right)$ is homotopy equivalent to $\Omega \operatorname{Map}\left(B, B_{Q} ; q_{*} k\right)$.

PROOF. In both cases one can prove, by obstruction arguments, that $\pi_{0}\left(\Pi_{p}^{q}\right)$ is surjective.

7. Some categorical considerations. In $\S 5$ we have shown that the construction of the homotopy equivalence $\delta_{p}$ is "natural" in a certain sense, but the use of this word would be somewhat vain if one could not justify it in terms of some categorical construction. This can in fact be done as we now indicate.

Let $B$ be a topological space having the same homotopy type as a CW-complex and define a category $\mathfrak{B}_{B}$ by taking as objects all pairs $(p, k)$ with $p: X \rightarrow B$ a numerable $G$-bundle (for some $G$ ) and $k: B \rightarrow B_{G}$ a classifying map for $p$. The morphisms from $(p, k)$ to $\left(p^{\prime}, k^{\prime}\right)$ are all pairs $(f, h)$ with $h: G \rightarrow G^{\prime}$ a continuous homomorphism such that $k^{\prime}=h_{*} k$ and $f: X \times_{G} G^{\prime} \rightarrow X^{\prime}$ a principal bundle isomorphism.

Let now $\mathfrak{S} \mathfrak{T} \mathfrak{O p}$ be the category of topological spaces and homotopy classes of continuous maps and consider the two functors $\Omega,(S): \mathfrak{B}_{\mathrm{B}} \rightarrow \mathfrak{F}_{\varepsilon} \mathfrak{T} \mathfrak{o p}$ defined as 
follows:

$$
\begin{aligned}
& \Omega(p, k)=\Omega \operatorname{Map}\left(B, B_{G} ; k\right) ; \quad \mathfrak{S}(p ; k)=\mathbb{S}(p), \\
& \Omega(f, h)=\Omega\left(h_{*}\right)_{B}: \Omega \operatorname{Map}\left(B, B_{G} ; k\right) \rightarrow \Omega \operatorname{Map}\left(B, B_{G^{\prime}} ; h_{*} k\right), \\
& \mathbb{S}(f, h)=f_{*} \Pi_{p}^{h}: \mathbb{S}(p) \rightarrow \mathbb{S}\left(p^{h}\right) \rightarrow \mathfrak{S}\left(p^{\prime}\right),
\end{aligned}
$$

where $f_{*}: \circlearrowleft \&\left(p^{h}\right) \rightarrow \circlearrowleft \&\left(p^{\prime}\right)$ is induced by $f$. One can easily check that both functors are well defined.

7.1 THEOREM. There exists a natural equivalence $\delta: \Omega \rightarrow$ (S) defined by the relation $\delta(p, k)=\left[\delta_{p}\right]: \Omega \operatorname{Map}\left(B, B_{G} ; k\right) \rightarrow \mathfrak{S}(p)$.

Proof. In the diagram

$$
\begin{aligned}
& \Omega \operatorname{Map}\left(B, B_{G} ; k\right) \stackrel{\delta_{p}}{\rightarrow} \quad H(p) \\
& \Omega\left(h_{*}\right)_{B} \downarrow \quad \downarrow \Pi_{p}^{h} \\
& \Omega \operatorname{Map}\left(B, B_{G^{\prime}} ; h_{*} k\right) \stackrel{\delta_{\left(p^{h}\right)}}{\rightarrow} \quad \leftrightarrow\left(p^{h}\right) \\
& 1 \downarrow \\
& \Omega \operatorname{Map}\left(B, B_{G^{\prime}} ; h_{*} k\right) \stackrel{\delta_{p^{\prime}}}{\rightarrow} \quad \mathfrak{S}\left(p^{\prime}\right)
\end{aligned}
$$

the upper square has been shown to be homotopy commutative in Theorem 5.3. The same technique used there can be applied to $f_{*}$ to prove the homotopy commutativity of the lower square. This, together with Theorem 4.3, suffices to prove the theorem.

REMARKS. (1) Both $\Omega \operatorname{Map}\left(B, B_{G} ; k\right)$ and $\mathscr{S}(p)$ are $H$-groups in the obvious way and it can be shown that the map $\delta_{p}$ is in fact an $H$-homomorphism (see [3, §4]). The functors $\Omega$ and (S) can therefore be viewed as taking values in the category of $H$-groups and homotopy classes of $H$-homomorphisms.

(2) The idea of having different objects of $\mathfrak{B}_{B}$ associated with the same bundle may not be very agreeable, as well as that of joining by a morphism only bundles whose chosen classifying maps are exactly matched by $h$. If we try to avoid this idea of "pointed" bundles we find out that $\Omega(p, k)$ is defined only up to homotopy type and so the only possible candidate for a range category is that of homotopy classes of $H$-groups and $H$-homomorphisms. With this choice the functors $\Omega$ and $\&$ become identical and we leave to the reader the decision on whether this gives rise to a more elegant or to a less meaningful result.

\section{REFERENCES}

1. P. I. Booth and R. Brown, Spaces of partial maps, fibred mapping spaces and the compact-open topology, General Topology Appl. 8 (1978), 181-195.

2. __ On the applications of fibred mapping spaces to exponential laws for bundles, ex-spaces and other categories of maps, General Topology Appl. 8 (1978), 165-179.

3. P. I. Booth et al.,H-spaces of self-equivalences of fibrations and bundles (preprint).

4. R. Brown and P. Heath, Coglueing homotopy equivalences, Math. Z. 113 (1970), 313-325. 
5. A. Dold, Partitions of unity in the theory of fibrations, Ann. of Math. (2) 78 (1963),223-255.

6. D. H. Gottlieb, Applications of bundle map theory, Trans. Amer. Math. Soc. 171 (1972), 23-50.

7. D. Husemoller, Fibre bundles, 2nd ed., Springer-Verlag, 1975.

8. I. M. James, The space of bundle maps, Topology 2 (1963), 45-49.

9. S. S. Koh, Note on the homotopy properties of the components of the mapping space $X^{S^{p}}$, Proc. Amer. Math. Soc. 11 (1960), 896-904.

10. J. Milnor, Construction of universal bundles. I, Ann. of Math. (2) 63 (1956), 272-284.

11. __ Construction of universal bundles. II, Ann. of Math. (2) 63 (1956), 430-436.

12. C. Morgan, F-fibrations and groups of gauge transformations, Ph.D. Thesis, Memorial Univ. of Newfoundland, 1980.

13. R. Schön, Fibrations over a CWh-base, Proc. Amer. Math. Soc. 62 (1977), 165-166.

14. E. Spanier, Algebraic topology, McGraw-Hill, 1966.

15. A. Trautman, Fibre bundles associated with space-time, Rep. Math. Phys. 1 (1970), 29-62.

16. _ On groups of gauge transformations, Lecture Notes in Physics, vol. 129, Springer-Verlag, 1980, pp. 114-120.

17. R. M. Vogt, Convenient categories of topological spaces for homotopy theory, Arch. Math. (Basel) 22 (1971), 546-555.

Department of Mathematics and Statistics, Memorial University of Newfoundland, St. John's, NewFoundLAND, A1B 3X7 CANADA

Department of Mathematics, University of British Columbia, Vancouver, British Columbia, VGT 1W5 CANADA (Current address) 